\title{
Água na Lua: o que sabemos hoje
}

\author{
Pedro Mota Machado \\ Instituto de Astrofísica e Ciências do Espaço e Faculdade de Ciências da Universidade de Lisboa \\ machado@oal.ul.pt
}

\begin{abstract}
Water in the Moon: what do we know today - Although the presence of water on the Moon today is indisputable, there are still several unanswered questions such as: what is the total amount of water in Moon's reservoirs?; what is its distribution, and in what form can we find it? In addition to addressing these issues, we also present here the methods and techniques that led to obtaining the scientific evidence related to the existence of water on the Moon.
\end{abstract}

Apesar da presença de água na Lua ser hoje indiscutível, ainda subsistem várias questões em aberto, nomeadamente: qual a quantidade total de água nos reservatórios na Lua?; qual a sua distribuição e em que forma a podemos encontrar? Para além de abordar estes temas, também são aqui apresentados os métodos e técnicas que conduziram à obtenção das evidências científicas relacionadas com a questão da existência de água na Lua.

A evidência da presença de água na Lua é hoje indiscutível. A quantidade e a sua distribuição na superfície e no subsolo lunar são questões que permanecem em aberto e a que urge dar uma resposta cabal.

A água existente hoje na Lua deverá ser de origem primordial (há cerca de 3-4 mil milhões de anos) e ter-se-á mantido até hoje fruto das características únicas da geometria com que a sua superfície é iluminada pela radiação solar [1]. Assim, nas altas latitudes, a radiação solar atinge a borda das crateras de forma rasante o que impede a sua penetração no interior das mesmas. Consequência deste défice de iluminação direta são os aglomerados de gelo de água detetados nas crateras lunares das regiões polares (Figura 1).
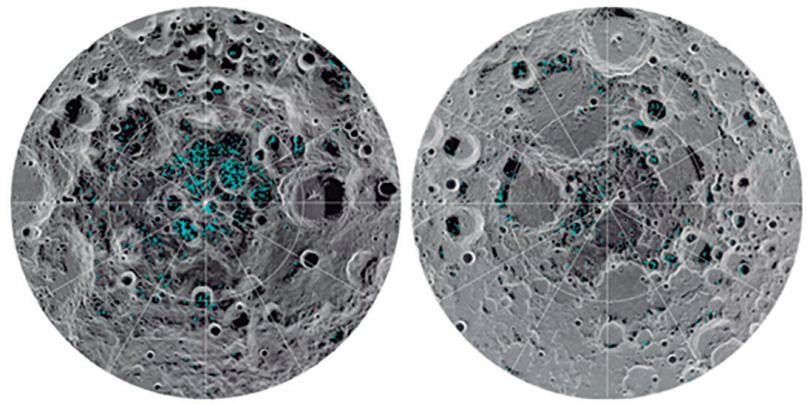

Figura 1 - Mapa com a distribuição de gelos de água presentes nas encostas sombrias de crateras nas regiões polares da Lua. Este mapa foi feito com base em dados obtidos pela missão espacial Chandrayaan-1.

Imagem: https://www.jpl.nasa.gov/news/news.php?feature=7218, créditos da imagem: NASA.

O contexto histórico da exploração lunar congrega missões americanas nos anos noventa do século XX, como são os casos das missões Clementine (NASA/USAF) e Lunar Prospetor (NASA), a que se seguiram missões da agência espacial europeia (ESA) e da sua congénere japonesa (JAXA), respetivamente SMART-1 e Kaguya. Mais recentemente, a Índia, com a sua missão Chandrayaan-1, a China, com a primeira fase da missão Chang'e [2], e os Estados Unidos da América, com a missão Lunar Reconnaissance Orbiter (NASA), contribuíram para a continuação da exploração espacial da Lua. A completar o ramalhete da recente exploração lunar por meio de missões espaciais dedicadas, temos a atual segunda fase da missão chinesa (Chang'e II) que dá pelo simpático nome de "Coelho de Jade". Por fim, há um neófito no restrito clube dos países que participaram na exploração espacial da Lua, a saber: Israel com a sua missão Beresheet e a sua malograda alunagem em abril de 2019.

Para além do gelo de água superficial detetado em crateras e fissuras, sobretudo nas altas latitudes lunares, há também confirmação da existência de água na exosfera lunar e abaixo da superfície [2,3]. A presença de água sub-superficial foi detetada a alguns metros de profundidade (2-5 m) através do estudo do material ejetado por impactos provocados na superfície.

A missão Chandrayaan-1 teve grande relevância na pesquisa da água na Lua. De facto, o seu foco de investigação e objetivo primordial centrou-se na deteção de evidências inequívocas da existência de água. Vários dos seus instrumentos foram desenhados e concebidos para esse fim. Para além de efetuar um mapeamento detalhado da topografia lunar e, consequentemente, do gelo de água superficial presente nas zonas sombrias das crateras lunares, as experiências científicas a bordo desta sonda espacial tinham como fito entender a génese e subsequente evolução mineralógica e química do nosso satélite natural. Outra investigação fundamental perpetrada por esta sonda consistiu na deteção e identificação de diversos componentes químicos presentes na rarefeita atmosfera lunar. $\mathrm{Na}$ verdade, o termo atmosfera é um abuso de linguagem para o caso da Lua. Dada a sua baixa força gravitacional, as parcas moléculas que por sublimação, ou devido a impactos com a superfície, se encontram na fase gasosa atingem muito rapidamente a velocidade de escape, perdendo-se no espaço. A atmosfera residual presente na Lua não está em equilíbrio hidrostático, sendo assim muito diáfana. É o que em geral se denomina uma exosfera.

A descoberta de água perto do polo Sul da Lua remonta a 1994 e ao legado da missão Clementine [4]. Esta descoberta foi possível por intermédio do uso de técnicas de ra- 
dar a varrer a superfície. Apesar de não ser possível detetar diretamente a presença de água usando radiofrequências, as propriedades refletivas do gelo de água podem ser encontradas através da emissão de radiação polarizada e na análise da sua reflexão em material transparente (gelo de água) presente na superfície lunar. O método baseia-se na emissão de ondas de rádio polarizadas (polarização circular direita) e na deteção do tipo de polarização com que a sua reflexão chega ao detetor. A razão entre as duas componentes de polarização na radiação detetada é utilizada para estudar a presença de meios transparentes a efetuar a reflexão das radiofrequências usadas.

A constatação mais recente da existência de água superficial baseou-se na utilização da experiência Moon Mineralogy Mapper (M3) a bordo da sonda Chandrayaan-1 $[5,6]$. Numa primeira fase foi detetada a assinatura espectral da água/gelo no infravermelho próximo (2-2,5 microns), tendo esta deteção sido efetuada na radiação solar refletida no lado diurno da superfície lunar. Numa segunda análise dos dados foi também detetada a "impressão digital" espetroscópica da presença de água em zonas escuras perto dos polos. A evidência assim detetada era muito ténue comparada com a primeira deteção, devido ao facto de a radiação agora estudada não provir de uma reflexão direta, mas sim de reflexões múltiplas nas encostas interiores das crateras a elevadas latitudes, que desse modo conseguiram transportar os indícios da presença de água nas zonas sombrias do interior das crateras até ao detetor M3. A equipa de investigadores desta missão conseguiu reunir evidências complementares às previamente citadas. Foram usados testes de laboratório para detetar padrões de absorção espetroscópica a 1,3, 1,5 e a 2 microns, os quais também estavam presentes nas observações da M3, sendo que estes padrões de absorção espectral constituem uma evidência clara da existência de gelo de água. Na verdade, a confirmação da existência de gelo de água superficial nas crateras a altas latitudes, e já muito perto dos polos lunares, veio dar mais consistência às deteções prévias de gelos perto dos polos pela missão Lunar Reconnaissance Orbiter (LRO). Os indícios da presença de gelo detetados pela missão LRO basearam-se em observações efetuadas com o seu altímetro laser (LOLA) e também com recurso a observações obtidas com o espetrofotómetro de ultravioleta (LAMP) a bordo da sonda LRO [7]. O espetrofotómetro UV usou as riscas espectrais Lyman-alfa, muito usadas na exploração de galáxias, para detetar os gelos de água nas crateras próximas dos polos.

No que toca à deteção de água sub-superficial, ela foi conseguida também por intermédio das observações efetuadas com radares de abertura sintética presentes tanto na missão Chandrayaan-1 como na LRO. Em ambos os casos, os mapas obtidos apresentam valores mais elevados da presença de água sub-superficial perto dos polos lunares.

O satélite LCROSS (Lunar CRater Observation and Sensing Satellite, no original), lançado em conjunto com o LRO em 2009, descobriu água nas regiões permanentemente sombrias da cratera Cabeus (perto do polo Sul) (Figura 2). Esta experiência baseou-se no despenhamento controlado de um módulo vazio do veículo lançador dessa missão (Centaur). O impacto produziu uma nuvem de detritos composta por duas plumas, uma formada por va- por e poeiras finas, ejetadas segundo um ângulo acentuado em relação ao solo, e a outra composta por partículas mais pesadas, ejetadas segundo um ângulo menor [8]. A interação do material ejetado com a radiação solar, e a absorção no infravermelho, foi analisada pelos sensores da LRO [9]. Este estudo revelou a presença tanto de vapor de água como de cristais de gelo de água, além de outros componentes minoritários como o monóxido de carbono, amoníaco, metano e uma pletora de moléculas orgânicas muito simples [10]. A experiência LCROSS detetou uma abundância relativa de água superior ao que anteriormente se suspeitava, atingindo um valor de percentagem em massa de 5,6 $\pm 2,9 \%$. Esta estimativa continua, ainda hoje, a ser um valor de referência [11].

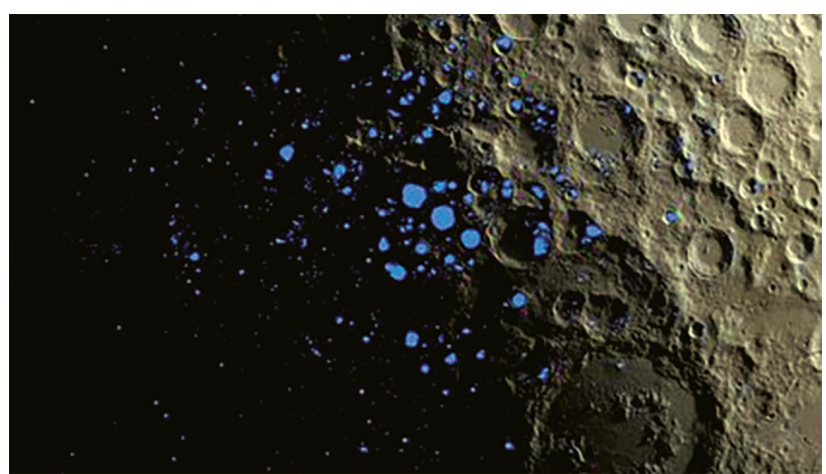

Figura 2 - As zonas permanentemente à sombra (a azul na imagem) cobrem cerca de 3\% da região polar Sul da Lua. Missão NASA Goddard/LRO. Imagem: https://svs.gsfc.nasa.gov/11230. Créditos da imagem: NASA's Goddard Space Flight Center.

As áreas de sombra permanente na Lua, como é o caso da cratera Cabeus, podem conter uma chave para a história e a evolução do sistema solar, da mesma forma que uma amostra de gelo terrestre revela dados antigos sobre a sua origem e evolução temporal.

O espectrógrafo Lyman Alpha Mapping Project (LAMP) a bordo da LRO, que opera no ultravioleta longínquo (100-200 nm), observou a pluma de material lunar ejetado pelo impacto da experiência LCROSS, centrando-se na análise da emissão da fluorescência da radiação solar ao interagir com o hidrogénio molecular $\left(\mathrm{H}_{2}\right)$ e monóxido de carbono. A curva de luz observada foi bem simulada pela expansão de uma nuvem de vapor, a cerca de $1000 \mathrm{~K}$, contendo aproximadamente $140 \mathrm{~kg}$ de hidrogénio molecular e $570 \mathrm{~kg}$ de monóxido de carbono [12]. Desta vez não foi detetada nenhuma evidência da molécula de água nesta gama espectral [10]. Com base neste detetor, a estimativa da presença de hidrogénio molecular na pluma de ejeção fixou-se nos 3,7\% em massa. Para dar algum contexto ao valor apresentado, repare-se que a concentração global média de hidrogénio no solo lunar é de cerca de 50 ppm, o que significa que o hidrogénio presente na pluma ejetada a partir da superfície encontra-se enriquecido por um fator de cerca de 740 vezes [13,14]. Por outro lado, a análise de voláteis feita no infravermelho próximo sugere abundâncias na ordem de 2 a 10\% em massa do hidrogénio presente de uma forma global, quer a nível atómico quer molecular $\left(\mathrm{H}_{2}, \mathrm{OH}\right.$ e $\left.\mathrm{H}_{2} \mathrm{O}\right)$. É de notar que o material ejetado proveio de uma profundidade de até $2-5 \mathrm{~m}$. 

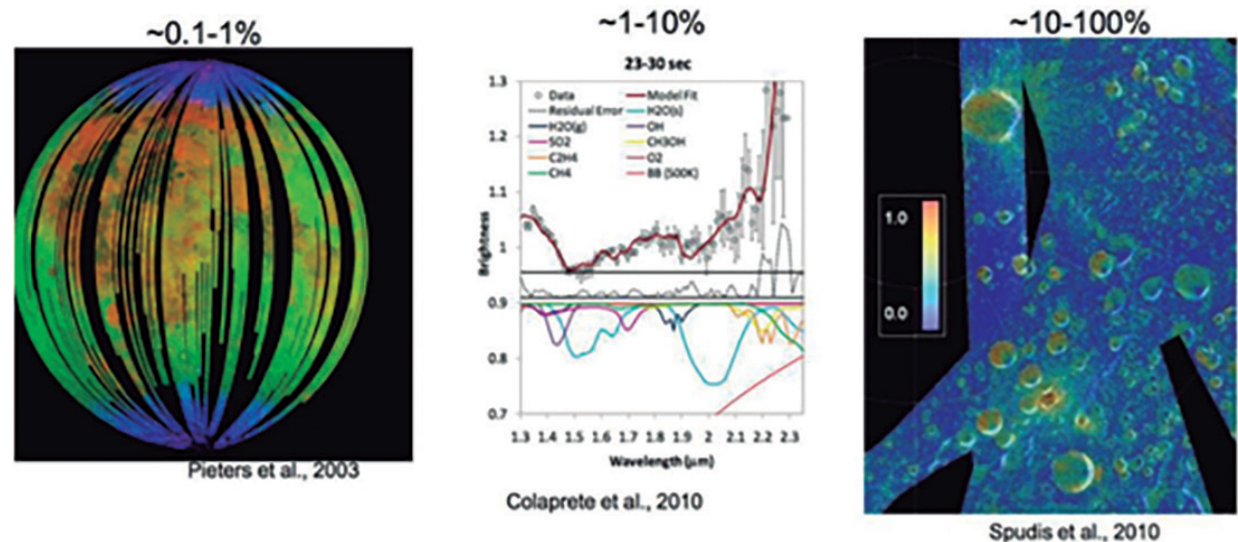

Figura 3 - À esquerda apresenta-se uma imagem da Lua obtida pelo instrumento Moon Mineralogy Mapper (Chandrayaan-1). As zonas a azul mostram a assinatura espectral de hidróxido no infravermelho próximo ( 3 microns), o que dá uma estimativa da existência de água nessas regiões. Na imagem central, devido à absorção no infravermelho, evidencia-se a presença de vapor de água, e de partículas de gelo de água, nas plumas de material ejetado pela experiência de impacto na superfície lunar LCROSS. À direita, resultados baseados na deteção de hidrogénio por espetrometria de neutrões na região polar Norte da Lua, missão Chandrayaan-1.

Imagem: https://www.airspacemag.com/daily-planet/how-much-water-moon-180967751/[10], créditos: SRO/NASA/JPL-Caltech/Brown Univ./USGS.

A investigação utilizando o infravermelho próximo foi realizada no decurso de três missões lunares (Chandrayaan-1, Cassini e EPOXI). Todas observaram uma banda de absorção perto dos 3 microns, que é gerada pela presença de água $\left(\mathrm{H}_{2} \mathrm{O}\right)$ e de hidroxilo $(\mathrm{OH})$ (Figura 3). A deteção foi efetuada a partir dos 60 graus de latitude e aumentando, paulatinamente, a intensidade da deteção em direção ao polo.

Outra técnica utilizada na pesquisa de água na Lua, mais propriamente da quantidade de hidrogénio, foi a espetrometria de neutrões. Esta técnica baseia-se na medição da quantidade e energia de neutrões, produzidos no espaço intergaláctico, após a sua interação com a superfície lunar, onde se dá uma absorção desses neutrões pelo hidrogénio presente no material do solo lunar. Assim, ao sondar a superfície lunar, um défice na deteção de neutrões tem uma elevada correlação com a presença de hidrogénio na zona estudada. O mapeamento de neutrões ao longo da topografia lunar mostra-nos as regiões ricas em hidrogénio, e porventura em água. Tanto a missão Lunar Prospector de 1998, como a missão mais recente $L R O$, tinham entre os instrumentos a bordo (payload) um espetrómetro de neutrões. Ambas as missões mediram valores consistentes da quantidade de hidrogénio nas regiões de elevadas latitudes e já perto dos polos. O valor obtido, perto de 150 ppm, é notoriamente mais elevado do que o que foi detetado entre as latitudes médias (cerca de 50 ppm).

Uma das limitações desta técnica assenta na sua resolução espacial, sendo da ordem da altitude a que o espetrómetro de neutrões faz o varrimento. Assim, a resolução espacial com que foi feita a deteção com este instrumento é de, na melhor das hipóteses, de 30 a 50 quilómetros. Por conseguinte, o valor de 1,5\% em massa obtido nos mapas gerados pelo espetrómetro de neutrões é um valor médio ao longo da superfície lunar, onde não estão resolvidas as crateras e não é claro o esperado valor mais elevado no seu interior.

Apesar das missões mais recentes terem reforçado as evidências prévias de existência de água na Lua, nomeadamente na forma de gelos nas encostas sombrias de crateras ou fissuras, principalmente nas regiões polares, ainda há muita controvérsia sobre a quantidade de água na Lua. Os diferentes métodos utilizados para detetar água na Lua deram resultados consistentes e permitiram uma validação cruzada, assente em técnicas independentes, da existência de água na Lua. A dúvida reside na precisão das deteções efetuadas, na abundância determinada pelas diferentes técnicas e na localização dos reservatórios de água.

Hoje ainda não temos uma resposta exata em relação à quantidade de água que existe na Lua. No entanto, uma estimativa conservadora aponta para um valor de cerca de cem milhões até mil milhões de toneladas presentes em cada uma das regiões polares [10]. O que ainda não se sabe, e que é preciso determinar, é, além da quantidade exata existente, a distribuição por estado físico e a distribuição entre a quantidade presente na forma de gelos superficiais e de água subterrânea. Outra questão em aberto prende-se com a sua proveniência: Qual a quantidade de água que tem uma origem primordial, datando da formação da Lua, e qual a quantidade que adveio da queda de meteoritos e de cometas? Um estudo baseado em medições in situ usando espetrómetros de massas de modo a determinar, sem ambiguidades, a razão isotópica dos constituintes da molécula de água, poderá trazer luz a este mistério. Além desta investigação ser relevante para o estudo per se deste corpo celeste, a sua inserção no estudo mais amplo da formação e evolução do sistema solar irá contribuir para conhecermos melhor o processo da sua formação e da sua história evolutiva.

Uma razão prática ligada à pertinência do estudo da existência, abundância e localização de água na Lua, assenta no recrudescimento do interesse em voltar a visitar a Lua [15]. Atualmente há missões a serem desenhadas pelas diversas agências espaciais, como a NASA (USA), a ESA (Europa), a JAXA (Japão), a China, a Índia e até Israel. A ideia de estabelecer colónias humanas de longa duração na Lua ganha cada vez mais importância, sendo exemplo disto o projeto Moon Village, da ESA. Para um projeto desta natureza, a presença de água é determinante $[10,16]$ pois além da sua relevância para consumo humano e para a eventual produção de alimentos localmente (estufas, culturas hidropónicas), a água poderá ser convertida nos seus 
constituintes, com o oxigénio a poder ser utilizado para dar viabilidade a habitats confinados e sustentáveis, e o hidrogénio e oxigénio como combustíveis para todo o tipo de atividades humanas na Lua, incluindo o seu uso como propelente de foguetões para a viagem de regresso à Terra.

\section{Referências:}

[1] A. Crotts, Astronomical Rev. 6 (2011) 4-20.

[2] R.N. Clark, Science 326 (2009) 562-564.

[3] J.W. Freeman, Jr., H.K. Hills, R.A. Lindeman, R.R. Vondrak, The Moon 8 (1973) 115-128.

[4] R.A. Simpson, G.L. Tyler, J. Geophys. Res. 104 (1999) 3845-3862.

[5] O.R. Green, C. Pieters, P. Mouroulis, M. Eastwood, J. Boardman, T. Glavich, et al., J. Geophys. Res. 116 (2011) E00G19.

[6] C.M. Pieters, J.N. Goswami, R.N. Clark, M. Annadurai, J. Boardman, B. Buratti, J.-P. Combe, M.D. Dyar, R. Green, J.W. Head, C. Hibbitts, M. Hicks, P. Isaacson, R. Klima, G. Kramer, S. Kumar, E. Livo, S. Lundeen, E. Malaret, T. McCord, J. Mustard, J. Nettles, N. Petro, C. Runyon, M. Staid, J. Sunshine, L.A. Taylor, S. Tompkins, P. Varanasi, Science 326 (2009) 568-572.

[7] P. Spudis, D.B.J. Bussey, S.M. Baloga, J.T.S. Cahill, L.S. Glaze, G.W. Patterson, R.K. Raney, T.W. Thompson, B.J. Thomson, E.A. Ustinov, JGR Planets 118 (2013) 2016-2029.

[8] A.P. Jordan, T.J. Stubbs, C.J. Joyce, N.A. Schwadron, H.E.
Spence, J.K. Wilson, JGR Planets 118 (2013) 1257-1264.

[9] K. Ennico, M. Shirley, A. Colaprete, L. Osetinsky, Space Sci. Rev. 167 (2012) 23-69.

[10] P. Spudis, Air \& Space Smithsonian, January 5, 2018.

[11] A. Colaprete, P. Schultz, J. Heldmann, D. Wooden, M. Shirley; K. Ennico, B. Hermalyn, W. Marshall, A. Ricco, R.C. Elphic, D. Goldstein, D. Summy, G.D. Bart, E. Asphaug, D. Korycansky, D. Landis, L. Sollitt, Science 330 (2010) 463-468.

[12] G.R. Gladstone, D.M. Hurley, K.D. Retherford, P.D. Feldman, W.R. Pryor, J.Y. Chaufray, M. Versteeg, T.K. Greathouse, A.J. Steffl, H. Throop, J.W. Parker, D.E. Kaufmann, A.F. Egan, M.W. Davis, D.C. Slater, J. Mukherjee, P.F. Miles, A.R. Hendrix, A. Colaprete, S.A. Stern, Science 330 (2010) 472-476.

[13] C.D. Neish, D.B.J. Bussey, P. Spudis, W. Marshall, B.J. Thomson, G.W. Patterson, L.M. Carter, J. Geophys. Res. 116 (2011) E01005.

[14] S. Nozette, P. Spudis, B. Bussey, R. Jensen, K. Raney, Space Sci. Rev. 150 (2010) 285-302.

[15] S. Li, P.G. Lucey, R.E. Milliken, P.O. Hayne, E. Fisher, J.-P. Williams, D.M. Hurley, R.C. Elphic, Nat. Acad. Sci. USA 115 (2018) 8907-8912.

[16] P.D. Spudis, "The value of the Moon: how to explore, live, and prosper in space using the Moon's resources”, Smithsonian Books, 2016.

\section{A luz como estímulo de catalisadores para a metátese de olefinas}

A metátese de olefinas é uma das ferramentas mais atrativas e poderosas para a formação de ligações $\mathrm{C}=\mathrm{C}$. Durante as últimas duas décadas, muitos esforços foram centrados no desenvolvimento de catalisadores de metais de transição mais eficientes. Uma atenção especial foi dedicada recentemente ao desenvolvimento de catalisadores de metátese latente, espécies inativas que necessitam de um estímulo externo para se tornarem ativas. Este tipo de catalisadores permite um melhor controlo sobre as reações. Vários estímulos têm sido explorados, incluindo calor, luz, ultrassons e comutação ácido/base e redox. A luz é, sem dúvida, o estímulo mais indicado, uma vez que é não-invasivo e pode ser facilmente manipulado. Têm sido publicados estudos sobre a metátese de olefinas promovida pela luz, mas abordam essencialmente a questão da ativação do processo e não tanto o seu controlo.

Tomislav Rovis e colegas da Universidade de Columbia, Nova Iorque, desenvolveram um sistema de metátese de olefinas controlada por luz visível que combina um pré-catalisador de ruténio contendo carbenos N-heterocíclicos (NHC) com catálise fotoredox. O catalisador, inativo nas condições ambientais, é ativado por luz visível azul na presença do fotocatalisador tetrafluoroborato de 2,4,6-trifenilpirílio. A oxidação do pré-catalisador leva à dissociação de um dos ligandos NHC e a subsequente reação de metátese. Várias reações foram testadas, incluindo metá-

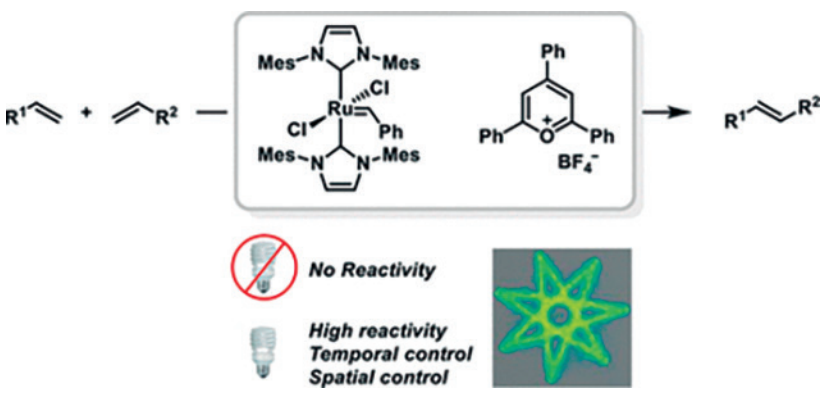
tese de fecho de anel (RCM) e metátese de abertura de anel (ROMP) tendo sido obtidos os produtos desejados com elevados níveis de resolução temporal e espacial e com bons a excelentes rendimentos. Segundo os autores, está aberta a possibilidade de aplicação em fotolitografia ou impressão 3D.

\section{Fonte:}

Olefin Metathesis Controlled with Visible Light, https://www.chemistryviews.org/details/news/11150294/Olefin_Metathesis_Controlled_with_Visible_Light.html (Acedido em 26/04/2019)

C. Theunissen, M.A. Ashley, T. Rovis, J. Am. Chem. Soc. 141 (2019) 6791-6796. 